\title{
Eplerenone in the Central Serous Chorioretinopathy Chronic
}

\author{
Mehdi Khamaily ${ }^{1,2, *}$, Joumany Brahim Salem ${ }^{1,2}$, Imane Tarib ${ }^{1,2}$, Wafaa Akioud ${ }^{1,2}$, \\ Soukaina Haddougui ${ }^{1,2}$, Yassine Mouzari ${ }^{1,2}$, Fouad El Asri ${ }^{1,2}$, Karim Reda ${ }^{1,2}$, \\ Abdelbarre Oubaaz ${ }^{1,2}$ \\ ${ }^{1}$ Department of Ophthalmology of the Mohammed V Military Teaching Hospital, Rabat, Morocco \\ ${ }^{2}$ Faculty of Medicine and Pharmacy, Mohammed V University, Rabat, Morocco
}

\author{
Email address: \\ mehdi.khamaily@gmail.com (M. Khamaily) \\ *Corresponding author
}

\section{To cite this article:}

Mehdi Khamaily, Joumany Brahim Salem, Imane Tarib, Wafaa Akioud, Soukaina Haddougui, Yassine Mouzari, Fouad El Asri, Karim Reda, Abdelbarre Oubaaz. Eplerenone in the Central Serous Chorioretinopathy Chronic. International Journal of Ophthalmology \& Visual Science. Vol. 5, No. 2, 2020, pp. 66-69. doi: 10.11648/j.ijovs.20200502.16

Received: May 21, 2020; Accepted: June 2, 2020; Published: June 17, 2020

\begin{abstract}
Summary: Central serous chorioretinitis is a relatively common eye condition. It is a maculopathy of the young subject, characterized by the presence of a retinal serous detachment, usually located at the posterior pole, associated with changes in the pigment epithelium. Materials and methods: a prospective study was conducted between Mars 2018 and Mars 2020 at the Mohammed V military hospital in Rabat, in 11 patients treated with eplerenone for a chronic CRSC evolving for at least 3 months with repercussions on visual acuity, at the dose of $25 \mathrm{mg}$ per day the first week, then $50 \mathrm{mg}$ per day for an average treatment duration of 15 weeks. For each patient, visual acuity and macular OCT (central thickness of the retina) were evaluated before treatment and at 1, 3 and 6 months. Results: The mean age of the patients was 32,54 $\pm 6,78$. 11 men. All patients were under stress, and just two patient reported the notion of taking corticosteroids. The average duration of progression of the pathology before treatment was 20 weeks. A clear improvement in visual acuity (logMAR) was observed in 9 patients: mean visual acuity increased from 0,7 at admission to 0,69 at 1 month (p: 0.014$), 0,4$ at 3 months (p: 0.013 ) and 0,28 at 6 months (p: 0.025 ). The mean central macular thickness decreased from $423 \pm 91 \mu \mathrm{m}$ before treatment, $317 \pm 82 \mu \mathrm{m}$ at 1 month and $292 \pm 73,6 \mu \mathrm{m}$ at 3 months and $251 \pm 94 \mu \mathrm{m}$ at 6 months (p 0.026, 0.01 and 0.038 respectively). 5 patients had a complete disappearance of SRF at 3 months. 1 other patients had a complete disappearance of the DSR at 6 months. The treatment was stopped for 3 patients, including 1 at 4 weeks for severe muscle cramps, and hyperkaliema for the 2 others at 10 weeks ans 11 weeks of treatment. Conclusion: In our study, the introduction of eplerenone resulted in significant improvement in both anatomical and functional outcomes in patients with chronic CSCR.
\end{abstract}

Keywords: Chronic Central Serous Chorioretinitis, Eplerenone, Sub-retinal Fluid, Mineralocorticoid Receptor

\section{Introduction}

Central serous chorio-retinopathy was first described in 1866 by Von Graefe, under the name of "recurrent central retinitis" [1]. With the fluorescein angiography, Gass showed in 1967 that there was a vanishing point in the retinal pigment epithelium (RPE) and a diffusion of the contrast agent in the sub-retinal space from the choroid [2]. He therefore hypothesized that RPE and the choroid were the tissues responsible for the disease, and used for the first time the term "central serous chorio-retininitis" to define it.

It was only later, once the hypothesis of inflammatory etiology was formally abandoned, that the term "central serous chorioretinopathy" was used to designate this pathology.

Many recent works have been interested in highlighting the probability of the role of hyperactivation of mineralocorticoid receptors at the level of the choroid vessels in the pathophysiology of central serous chorioretinopathy, based on this theory, we were interested in eplerenone, an anti aldosterone, a drug class widely used in cardiology, 
which inhibits the mineralocorticoid pathway, and therefore according to this theory act directly on one of the pathophysiogenicity pathways.

\section{Material and Method}

We conducted a prospective study in the ophthalmology department of the military instruction hospital Mohammed V of rabat, including 11 cases of chronic central serous chorioretinopathy, between March 2018 and March 2020.

The eplerenone or INSPRA ${ }^{\circledR}$ is a mineralocorticoid receptor antagonist used off-label for this indication, informed consent was signed by all of our patients after explaining the entire protocol, alternatives, benefits and risks of this treatment.

We analyzed the data of the consultations at inclusion, 1 month (M1), 3 months (M3) and 6 months (M6) for each patient. During each of these consultations, we listed for each patient, the tolerance to treatment, the best visual acuity corrected by far on the Monoyer scale converted to Log MAG, the foveal thickness in high definition OCT (in microns), the size of the serous retinal detachment in OCT (in microns).

The first endpoint was primarily functional, namely the improvement of the corrected visual acuity, as well as from an anatomical point of view, in particular the central macular thickness, the size of the SRF and the choroidal thickness at the OCT. not to mention tolerance to eplerenone.

We included in our study, any central serous chorioretinopathy that exceeds 3 months with a SFR and a significant visual impact.

We first excluded the contraindications of Eplerenone, namely the pregnant woman, and any patient with a history of renal failure or serum potassium disorder.

Patients with associated macular pathology

History of previous treatments (focal laser, PDT dynamic phototherapy, injection of anti-VEGF).

On optical coherence tomography, we measured two parameters:

1. On a high resolution line: the central macular thickness integrating the space between the retinal pigment epithelium and the internal limiting membrane, performed automatically by the OCT software

2. On a high resolution line: the height of the retinal serous detachment, measured manually with a calliper between the pigment epithelium and the line of external articles of photoreceptors.

\section{Results}

We studied 11 eyes of 11 patients who presented with clinically significant central serous chorioretinopathy more than 3 months.

Our 11 patients were male, with an average age of 32.54 years $+/-6.78$ (26 to 47 years). In all the patients in our series, the state of professional or personal stress was present, with the corticotherapy taken in 2 patients as well as the active smoking in 6/11 (Figure 1).

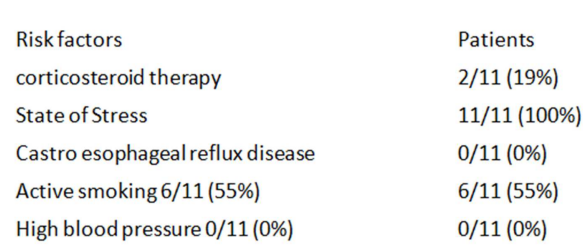

Figure 1. Risk factors in the patients in our study.

The decrease in acuity was the common symptom of all the patients with an initial mean on the Monoyer scale of $2.18 / 10(\log \mathrm{MAR}=+0.7 \pm 0.21)$ with extremes of $1 / 10$ $(\log$ MAR +1$)$ to $4 / 10(\log$ MAR +0.4$)$, metamorphopsia was found in 5 patients, a micropsy in 2 patients with a central scotoma in 5 patients, with an average duration of symptoms of 20 weeks (5 month) (Figure 2).

\begin{tabular}{lll} 
Symptoms & \multicolumn{2}{l}{ Patients } \\
Impaired visual acuity & $11 / 11$ & $(100 \%)$ \\
Metamorphopsis & $5 / 11$ & $(46 \%)$ \\
Micropsis & $2 / 11$ & $(19 \%)$ \\
Central scotoma & $5 / 11$ & $(46 \%)$
\end{tabular}

Figure 2. The most encountered symptoms in our study.

Eplerenone (INSPRA $\AA$ ), which is available as a $25 \mathrm{mg}$ tablet, was prescribed at a dose of $25 \mathrm{mg} /$ day for the first week, then $50 \mathrm{mg} /$ day for a minimum duration of 1 month, with an average duration of treatment. 15 weeks (between 1 month and 4 months).

The treatment was prolonged according to the anatomical and functional evolution, the tolerance of the side effects and the results of the serum potassium which was measured on Day 0, D7, at 1 month and then every month while the patient is under treatment of a systematically, and each time the side effects worsen in the patient.

The average duration of treatment was 15 weeks (between 1 month and 4 months)

Side effects to treatment were noted, including diarrhea in 6 patients, tolerable muscle cramps in 5 patients, followed by low back pain in 3 with one case of insomnia. suppressed by symptomatic treatment. (Figure 3)

$\begin{array}{lll}\text { Side effects } & \text { Patients } & \\ \text { diarrhea } & 6 / 11 & (55 \%) \\ \text { Muscle cramps } & 5 / 11 & (46 \%) \\ \text { Lower back pain } & 3 / 11 & (28 \%) \\ \text { Insomniea } & 1 / 11 & (9,1 \%) \\ \text { Hyperkaliémia } & 2 / 11 & (19 \%)\end{array}$

Figure 3. The main side effects in patients in our Study.

In addition, immediate discontinuation of treatment was necessary in 3 patients, including 1 to 4 weeks of treatment for severe muscle cramps, and hyperkalaemia in the other 2 at 10 weeks and 11 weeks of treatment.

Our results concern the best visual acuity corrected in far vision on the Monoyer scale, covert in Log MAR, the central foveal thickness and the size of the SFR at macular OCT.

There was a statistically significant improvement in the M1 AVCM (0.62 \pm 0.19$) \operatorname{LogMAR} ; \mathrm{p}=0.014)$, M3 (0.41 \pm 0.14 LogMAR; $p=0.013)$ and M6 (0.28 \pm 0.14 LogMAR, $p$ $=0.025)$ (Figure 4) 


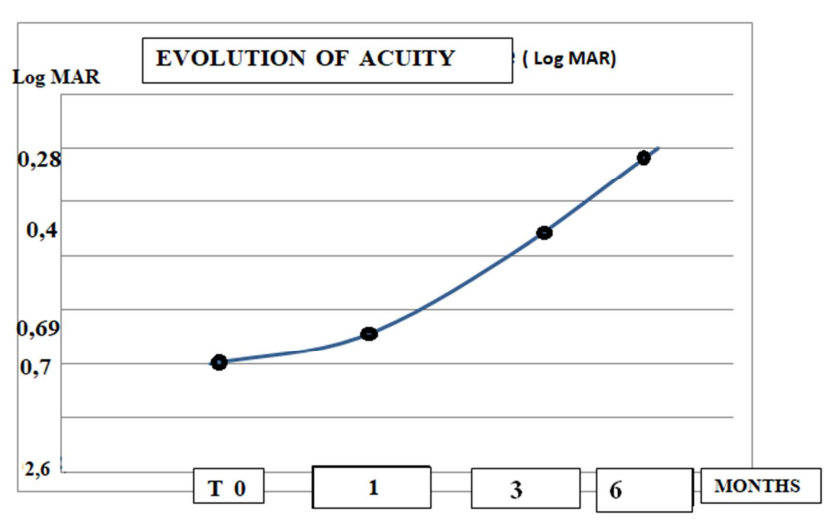

Figure 4. The best visual acuity corrected in Log MAR.

Concerning the RSD, the central mean central macular thickness in a non-statistically significant manner at M1 (317 \pm 82 microns; $p=0.066)$ then statistically significant at M3 (292 \pm 73.6 microns; $\mathrm{p}=0.01)$ and M6 (251 \pm 94 microns; $\mathrm{p}=0.038)$. (figure 5). Similarly, there was a non-statistically significant decrease in its size at M1 (109 \pm 80.9 microns; $p=0.059)$ and which became significant at M3 (73 \pm 33.4 microns; $p=0.009)$ and M6 (24 \pm 18.2 microns; $p=0.006)$. (figure 6)

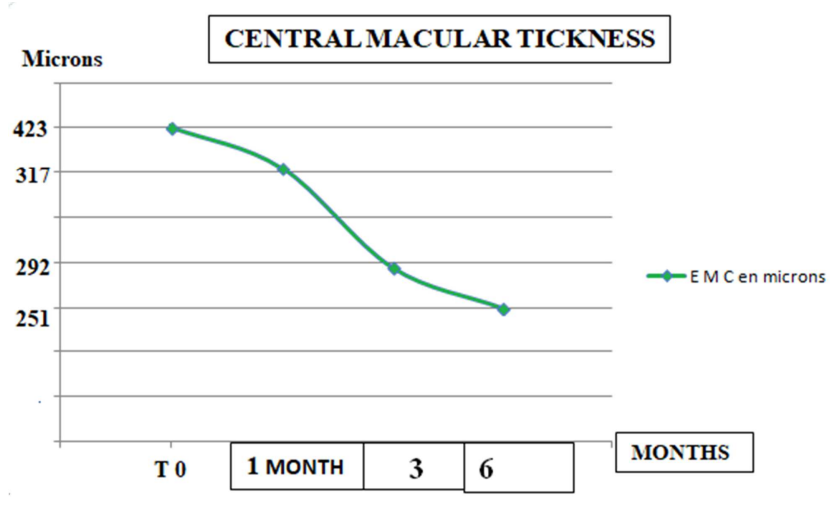

Figure 5. The evolution of the central macular thickness.

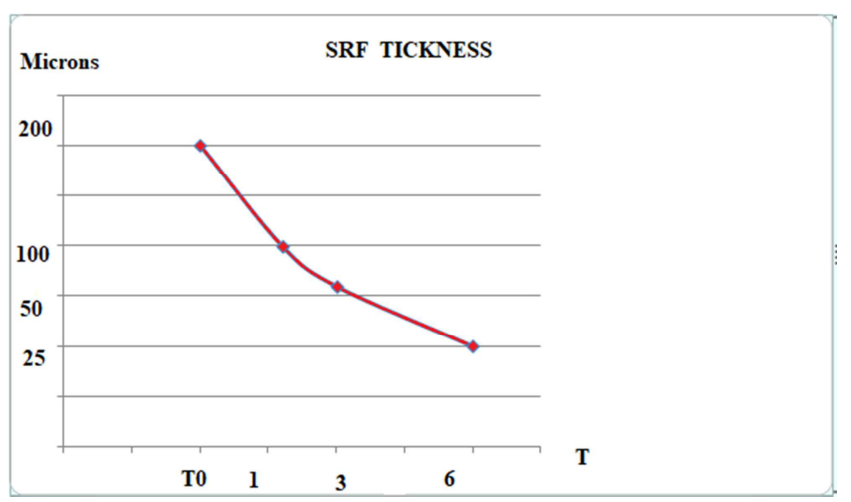

Figure 6. The evolution of the SRF.

The total drying of the SRF was noted that from the 3rd month of treatment in 5 patients, to 6 months in 1 patient, as for 1 patient, it kept a discreet SRF.

We report a case from our series, a 36-year-old patient, who presented with a well-developed chronic CRSC on Eplerenone. (Figure 7)

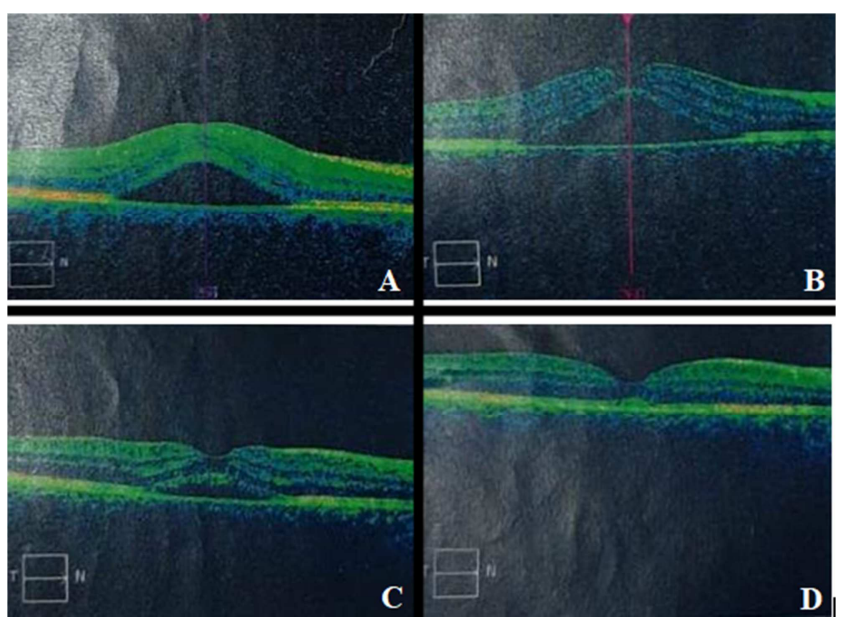

Figure 7. A 36-year-old patient, who presented with a well-developed chronic CRSC on Eplerenone.

A: SRF with EMC at 558 microns before treatment

B: Slight decrease in MCT to 547 microns at M1 processing.

C: Clear decrease in size of DSR and EMC to 358 microns at M3.

D: Normal CMT with total drying of the SFR to M6.

\section{Discussion}

We were interested in eplerenone in our study, an antialdosterone that binds and inhibits the mineralocorticoid receptors. This molecule is better tolerated than spironolactone according to Rubiano [3] it is used mainly in cardiology in heart failure and myocardial infarction. A first pilot study in 2013 by Bousquet evaluates eplerenone in the treatment of refractory CRSCs [4]. Of the 13 patients treated, $69 \%$ had a complete resolution of the DSR at 3 months, with no major side effect reported. This study was an extension of work on the eyes of rats carried out by the same team [5]

All the patients in our series were male with an average age of 32.5 years. In the various series in the literature, a clear predominance of men was noted with an average age which varied between 40.7 [6] years and 54.2 [4] years depending on the studies.

In most studies, taking corticosteroid therapy is known as a major risk factor whatever its dosage form, sometimes even at low doses, Asensio-Sánchez VM published in January 2020 a case of bilateral central serous chorioretinopathy, 1 month after a Intra-tarsal injection of Triamcinolone for a chalazion, the CRSC spontaneously resolved well 3 months after diagnosis [7]

Nowadays, the definition of persistent CSCR is debatable, but it is most often defined as a DSR lasting more than 3 months or recurrence of the disease within one year [8]

The period of persistence is variable according to the authors (between 3 and 6 months), but the most cited periods are 3 and 4 months.

In the Lotery study, the overall incidence of events described with eplerenone (53\%) was similar to that observed with placebo (54\%). The rate of discontinuation due to adverse events was $4.4 \%$ in patients treated with 
eplerenone and $4.3 \%$ in patients treated with placebo. The common $(>1 / 100)$ or serious adverse events observed in this study for which a relationship with treatment was suspected and whose incidence was greater than that observed with placebo [9]

Another prospective study in Boston conducted in 2019 also by Moein et al on 13 patients with the diagnosis of chronic CSCR. All patients received $50 \mathrm{mg} /$ day eplerenone for 4 weeks. The best corrected visual acuity and OCT parameters, including subretinal fluid, choroidal thickness and central macular thickness, were measured manually. The mean height of the DSR decreased slightly after one month's follow-up from the initial value, but the change was not statistically significant $(94.18 \pm 17.53$ versus $113.15 \pm 18.69 ; \mathrm{p}=0,08)$. The subfoveal choroidal thickness and the central macular thickness were significantly reduced compared to the baseline $(6.6 \%$ [p $=$ $0.002]$ and $7.05 \%[p=0.04]$, respectively). The final corrected visual acuity did not change significantly $(20 / 28$ versus $20 / 30[p=0.16]$ ) [8]

\section{Conclusion}

These encouraging results allow us to integrate eplerenone into the already existing therapeutic arsenal, however, randomized double-blind studies remain necessary in order to establish a standardized therapeutic protocol specifying the dosage and the optimal duration of treatment.

\section{Conflict of Interest}

The authors declare that they have no competing interests.

\section{References}

[1] Von Graefe A. - Ueber zentrale recidivierende Retinitis. Graefes Arch Clin Exp Ophthalmol 1866; 12: 211-215.

[2] Gass JD, Norton EW, Justice J. Serous detachment of the retinal pigment epithelium. Trans - Am Acad Ophthalmol Otolaryngol Am Acad Ophthalmol Otolaryngol. Déc 1966; 70 (6): 990-1015.

[3] William Fusi-Rubiano Oral medications for central serous chorioretinopathy: a Literature Review The Royal College of Ophthalmologists 2019.

[4] Bousquet E, Beydoun T, Zhao M, Hassan L, Offret O, BeharCohen F. Mineralocorticoid receptor antagonism in the treatment of chronic central serous chorioretinopathy: a pilot study. Retina Phila Pa. déc 2013; 33 (10): 2096-102.

[5] Daruich A, Matet A, Dirani A, Bousquet E, Zhao M, Farman $\mathrm{N}$, et al. Central serous chorioretinopathy: Recent findings and new physiopathology hypothesis. Prog Retin Eye Res. sept 2015; 48: 82-118.

[6] Omer Farooq et al Effect of oral eplerenone in anatomical and functional improvement in patients with chronic central serous chorioretinopathy Pak J Med Sci. 2019; 35 (6): 1544-1547.

[7] Asensio-Sánchez et al. Bilateral and multifocal central serous chorioretinopathy after injecting triamcinolone into a chalazion. Epub 2020 Mar; 95 (3): 141-145.

[8] Hamid reza Moein et al. Short-term eplerenone for treatment of chronic central serous chorioretinopathy; a prospective study. Int J Retin Vitr 2019. 5: 39.

[9] Andrew Lotery et al. Eplerenone for chronic central serous chorioretinopathy in patients with active, previously untreated disease for more than 4 months (VICI): a randomised, doubleblind, placebo-controlled trial. Lancet 2020; 395: 294-303. 